\title{
Beurteilungsspielräume als Wissensproblem - am Beispiel Regulierungsverwaltung
}

\author{
Wolfgang Schulz*
}
A. Einführung
330
B. Lehre vom Beurteilungsspielraum..... 331
I. Begründungen .................... 331
II. Normative Ermächtigung der Ver-
waltung .......................... 333
1. Fallgruppen .................. 334
2. Vorgehen der Rechtsprechung am Beispiel des $\$ 10$ TKG ...... 336

C. Wissensverarbeitung und Entscheidungsqualität ...................... 338

I. Differenzierung von Wissen......... 339

1. Implizites und explizites Wissen ......................... 339

2. Wissen und Nichtwissen ........ 341
3. Verwaltungsentscheidungen und gerichtliche Kontrolle in der Wissensperspektive............... 342

II. Zu den informationalen Strukturmerkmalen von Entscheidungen .... 343

1. Typ 1: Unhintergehbare Flüchtigkeit von Wissen .................. 343

2. Typ 2: Notwendigkeit diskursiver Produktion von Entscheidungswissen ................... 345

3. Typ 3: Die Notwendigkeit impliziten Wissens ................... 346

D. Fazit........................... 349

Die Lehre von den Beurteilungsspielräumen hat an einigen Stellen Struktur gewonnen, zeichnet sich aber an anderen noch durch eine gewisse Beliebigkeit aus. Der Beitrag untersucht, inwiefern die Frage der Wissensverarbeitung eine Rolle dafür spielt, dass intendiert vage Begriffe von Gerichten nur begrenzt überprüft werden können. Zur Illustration werden Entscheidungsstrukturen im Bereich der Regulierungsverwaltung, etwa im Telekommunikationssektor, herangezogen. Davon ausgehend wird ein Typ von Beurteilungsspielräumen konturiert.

\section{A. Einführung}

Potter Stewart vom US Supreme Court ist mit einem Satz zur Konkretisierung eines unbestimmten Rechtsbegriffs berühmt geworden: „I know it when I see it. “1 Dieser Beitrag will die Perspektive für Rechtsprechung und Verwaltung umdrehen und nach Gegenständen fragen, für die gilt: „I see it when I know it“.

Der moderne Staat ist eine wissensbasierte Organisation. ${ }^{2}$ Schon daher erscheint es sinnvoll, Institute wie den des Beurteilungsspielraums aus der Wissensperspektive zu betrachten. Die normative Ermächtigungslehre, die sich in Varianten durchgesetzt hat, nimmt bereits implizit auf Wissen und Information Bezug. Der Beitrag soll zei-

* Der Autor ist Direktor des Hans-Bredow-Instituts für Medienforschung und Professor für Medienrecht und Öffentliches Recht einschließlich ihrer theoretischen Grundlagen an der Universität Hamburg; der Beitrag basiert auf einem Habilitationsvortrag.

1 Stewart Potter in der Entscheidung Jacobellis v. Ohio 378 U.S. 184 (1964).

2 A. Voßkuble, Das Konzept des rationalen Staates, in: G.F. Schuppert/A. Voßkuhle (Hrsg.), Governance von und durch Wissen, Baden-Baden 2008, S. 13 (16); vgl. auch einführend zu dieser Perspektive: T. Horstmann, Die Vergrößerung und Verschönerung des Käfigs: Der Staat als wissensbasierte Organisation, zu finden unter: www.humboldt-forum-recht.de Jahrgang 2001, Beitrag 9. 
gen, dass ein expliziter und differenzierter Blick auf die Wissensverarbeitung im Verhältnis Behörde und Gerichte hilfreich ist, um noch bestehende Probleme zu lösen.

\section{B. Lehre vom Beurteilungsspielraum}

\section{Begründungen}

Die Frage nach dem Beurteilungsspielraum kann bei der Normanwendung auf zwei Ebenen gestellt werden, die - wie etwa von $\mathrm{Koch}^{3}$ formuliert - als normtheoretisch und staatstheoretisch bezeichnet werden können. Zunächst stellt sich auf Ebene des Normtextes die Frage nach Unterschieden, die im Hinblick auf die Kontrollfunktion relevant sind. Jeder Rechtsbegriff ist auslegungsbedürftig und insofern nicht voll bestimmt, als es der Durchführung eines methodisch gesteuerten Prozesses bedarf, seine Begriffsgrenzen für die Rechtsanwendung zu markieren.

Unklar ist immer noch, ob ein Kontinuum der Vagheit von Begriffen zugrunde zu legen ist oder sich kategoriale Unterschiede ergeben, so dass „unbestimmte Rechtsbegriffe" als eigene Gruppe erscheinen. In der Semantik werden durchaus Präzisierungen versucht. So wird wie folgt unterschieden: ${ }^{4}$ Vagheit kann etwa durch Relativausdrücke entstehen. Diese sind immanent vage; in Rechtstexten beliebte Ausdrücke wie „wesentliche Abweichung“ oder „geringfügige Unterschreitung“ gehören dazu. Zudem werden Begriffe mit lexikalischer Vagheit ausgemacht und derart vage Begriffe als unexakte Mengen definiert, die einen Kern eindeutig zuordbarer Objekte enthalten und eine Penumbra von Objekten, deren Zuordnung unklar ist. Für die Entwicklung von Systemen künstlicher Intelligenz ist dieser Unsicherheitsbereich trotz aller „Fuzzy Logic“ noch immer ein Hauptproblem. ${ }^{5}$

In der natürlichen Sprache ist Vagheit kein Mangel, sondern Mittel sprachlicher Ergonomie - sie wird vom Sprecher zuweilen gezielt eingesetzt. ${ }^{6}$ Auch beim Gesetzgeber als Sprecher kann man daher nach der Explizitheit der Vagheit fragen: Ergibt sich aus der Auslegung der einschlägigen Norm, dass der Gesetzgeber nicht nur eine, sondern unterschiedliche Auslegungsergebnisse als richtig markieren wollte? In diesem Falle liegt ein intendiert vager Rechtsbegriff vor. Da die normative Ermächtigungslehre davon ausgeht, dass der Gesetzgeber eine Entscheidung über die Letztentscheidungskompetenz getroffen hat, liegt es nahe, nur bei intendiert vagen Rechtsbegriffen Beurteilungsspielräume für eröffnet zu halten.

3 H.-J. Koch, Unbestimmte Rechtsbegriffe und Ermessensermächtigungen im Verwaltungsrecht, Frankfurt am Main 1979, S. $16 \mathrm{f}$.

4 Vgl. hierzu: M. Pinkal, Vagheit und Ambiguität, in: A. von Stechow/D. Wunderlich (Hrsg.), Semantik: Ein internationales Handbuch zeitgenössischer Forschung, Berlin u.a. 1991, S. 250 (251 ff.).

5 C. Borgelt/H. Timm/R. Kruse, Unsicheres und vages Wissens, in: G. Görz/C.-R. Rollinger/J. Schneeberger (Hrsg.), Handbuch der künstlichen Intelligenz, 4. Aufl., München u.a. 2003, S. 291 (293 ff.).

6 Etwa D. Wunderlich, Arbeitsbuch Semantik, 2. Aufl., Frankfurt am Main u.a. 1991, S. 74. 
In diesen Fällen rückt die Garantie effektiven Rechtsschutzes aus Art. 19 Abs. 4 GG sowie das Rechtsstaats- ${ }^{7}$ und, jedenfalls implizit, auch das Demokratieprinzip ${ }^{8}$ in den Vordergrund. ${ }^{9}$ Dem klassischen Verständnis - das hier nicht hinterfragt wird zufolge soll in jeder Verwaltungsentscheidung der Wille des demokratisch legitimierten Gesetzgebers realisiert werden. Die Prüfung der Gerichte bezieht sich daher (auch) auf die Einhaltung dieser Bindung an das Gesetz. ${ }^{10}$ Auslegung und Anwendung unbestimmter Rechtsbegriffe unterliegen der traditionellen Lehre folgend deshalb grundsätzlich in vollem Umfang der gerichtlichen Überprüfung. ${ }^{11}$

Als gegenläufiges verfassungsrechtliches Prinzip erweist sich das der Gewaltenteilung und -zuordnung ${ }^{12}$ sowie wiederum das Rechtsstaatsgebot, die eine funktionsgerechte Aufgabenzuweisung fordern. Dies besagt im Kern, dass Entscheidungen im Staatsgefüge von dem Organ zu treffen sind, das für die Entscheidung am besten geeignet erscheint. ${ }^{13}$ Es geht letztlich um die Qualität staatlichen Entscheidens und damit um den Topos der Entscheidungsrichtigkeit. ${ }^{14}$ Vor diesem Hintergrund kann einer ge-

7 S. Kautz, Absprachen im Verwaltungsrecht, Berlin 2002, S. 134 f.; im Überblick H. Schulze-Fielitz, in: H. Dreier (Hrsg.), Grundgesetz- Kommentar, Bd. I, 2. Aufl., Tübingen 2004, Art. 19 Rn. 116; vgl. allgemein zu den rechtlichen Grenzen bei Beurteilungs- und Ermessenspielräumen: Kopp/Schenke, VwGO Kommentar, 18. Aufl., München 2012, $\mathbb{1} 14$ Rn. 40; D. Ehlers, Staatliche Verwaltung, in: H.-U. Erichsen/D. Ehlers (Hrsg.), Allgemeines Verwaltungsrecht, 14. Aufl., Berlin u.a. 2010, $\mathbb{1}$ Rn. 48.

8 G. Kirchhof, Die Allgemeinheit des Gesetzes, Tübingen 2009, S. 302 m.w.N.

9 H. Rossen-Stadtfeld, Beurteilungsspielräume der Medienaufsicht, ZUM 2008, S. 457 (461); vgl. auch zu den verfassungsrechtlichen Anforderungen an unbestimmte Rechtsbegriffe: S. Brunner, Beurteilungsspielräume im neuen Jugendmedienschutz - eine nicht mehr vorhandene Rechtsfigur?, Münster 2005, S. $33 \mathrm{ff}$.

10 stRspr., BVerfGE 15, 275 (282); E 18, 203 (212); E 51, 304 (312); E 101, 106 (123); Schulze-Fielitz (Fn. 7), Art. 19 Rn. 87; M. Sachs, in: Ders., Grundgesetz Kommentar, 6. Aufl., München 2011, Art. 19 Rn.145; P. Badura, Grenzen und Alternativen des gerichtlichen Rechtsschutzes in Verwaltungsstreitsachen, JA 1984, S. 83 (90).

11 So bereits BVerfGE 7, 129 (154); E 13, 153 (164); E 56, 257 (275);E 103, 142 (156); BVerfG, Beschluß vom 28.6.1983 - 2 BvR 539, 612/80 = NJW 1984, S. 33 (35); BVerwGE 15, 207 (208); E 100, 221 (225); E 108, 190 (197 f.); E. Schmidt-Aßmann, in: F. Schoch/E. Schmidt-Aßmann/R. Pietzker (Hrsg.), VwGO Kommentar, München, Lsbl. Stand 2010, Einleitung Rn. 183, 186; A. Decker, in: H. Posser/ H. A. Wolff (Hrsg.), VwGO Kommentar, BeckOK 2011, $\mathbb{S} 114$ Rn. 32 ff.; a.A.: Sommer/LübbeWolff, in: BVerfGE 108, 129 (145); zu Grenzen richterlicher Fehlersuche: BVerwGE 116, 188 (196f.).

12 BVerfGE 79, 106 (120); E 80, 103 (108); E 81, 70 (88); E 82, 209 (224f.); w.N. bei E. Pache, Tatbestandliche Abwägung und Beurteilungsspielraum, Tübingen 2001, S. 36, Brunner, Beurteilungsspielräume im Jugendmedienschutz (Fn. 9), S. 33 ff.

13 Zur sog. funktionsgerechten Organstruktur: O. Küster, Das Gewaltproblem im modernen Staat, AöR 75 (1949), S. 397 (402 ff.); vgl. das Urteil des BVerfG zur Raketenstationierung: BVerfGE 68, 1 (86); E 95, 1 (15); E 98, 218 (251f.); T. von Danwitz, Der Grundsatz funktionsgerechter Organstruktur, Der Staat Bd. 35, S. 329 (330); später: Sachs (Fn. 10), \$20 Rn. 81; H. Schulze-Fielitz, in: H. Dreier (Hrsg.), Grundgesetz-Kommentar, Bd. II, 2. Aufl., Tübingen 2007, Art. 20 Rn. 108; P. Lerche, Gewaltenteilung - deutsche Sicht, in: J. Isensee (Hrsg.), Gewaltenteilung heute, Heidelberg 2000, S. 75 (78); F. Ossenbühl, Aktuelle Probleme der Gewaltenteilung, DÖV 1980, S. 548, (551); K. Hesse, Grundzüge des Verfassungsrechts der Bundesrepublik Deutschland, Neudr. d. 20.Aufl., Heidelberg 1999, Rn. 482, 484; W. Schmitt Glaeser, Der freiheitliche Staat des Grundgesetzes: Grundzüge, Tübingen 2008, S. 274; S. F. Schulz, Reprogenetik zwischen Forschungsfreiheit und Freiheitsstrafe, Berlin 2007, S. 312 .

14 von Danwitz, Organstruktur (Fn. 13), S. 336; zur Bedeutung dieses Topos: W. Hoffmann-Riem, Reform des Allgemeinen Verwaltungsrechts: Vorüberlegungen, DVBl. 1994, S. 1382 (1382). 
setzlichen Ermächtigung der Verwaltung zur Letztentscheidung die Einschätzung des Gesetzgebers zugrunde liegen, durch diese Funktionszuweisung die Entscheidungsqualität zu erhöhen, etwa auch, um gegenläufige grundrechtlich geschützte Interessen in ein verfassungskonformes Verhältnis zu bringen. Die Analyse von Beurteilungsspielräumen sollte also im Zusammenhang mit Qualitätsstandards von Entscheidungen vorgenommen werden. Da die Qualität einer Verwaltungsentscheidung sich dabei nicht in ihrer Rechtmäßigkeit erschöpft, ${ }^{15}$ die Verwaltungsgerichtsbarkeit aber auf deren Kontrolle programmiert ist, kann die Qualität der Entscheidung durch gerichtliche Kontrolle verändert werden; jedenfalls wenn das Gericht die Gestaltungsfunktion der Verwaltungsentscheidung nicht vollständig substituieren kann. Dabei wird zunehmend auch ein Eigenbereich der Verwaltung anerkannt. ${ }^{16}$

Die Kontrolle von Verwaltungsentscheidungen beeinflusst unvermeidlich die Gestaltungsfunktion der Verwaltungsentscheidung. Gestaltung durch die Verwaltung und Kontrolle durch die Gerichte mögen zwar in zunehmend multipolaren und daher unübersichtlichen Regulierungslagen nur noch schwer auseinanderzuhalten sein, bleiben aber analytisch unterscheidbar. ${ }^{17}$

Besondere Bedeutung kommt der Grenzziehung zwischen Gestaltung und Kontrolle dann zu, wenn die Verwaltung als Organisation für die zu bearbeitende Gestaltungsaufgabe entweder besser als das Gericht oder sogar allein qualifiziert erscheint und daher zur Letztentscheidung ermächtigt wurde. Neben Ermessensregelungen ist es gerade die durch unbestimmte Rechtsbegriffe geprägte Programmierung, die Abgrenzungsschwierigkeiten hervorruft. ${ }^{18}$

\section{Normative Ermächtigung der Verwaltung}

Im Falle unbestimmter Rechtsbegriffe muss ein spezifischer Grund dafür gegeben sein, dass und inwieweit hier gerade die Verwaltung und nicht das Verwaltungsgericht das letzte Wort hat, unter den unterschiedlichen Optionen, die das Gesetz für die Entscheidung eröffnet, auszuwählen. Inwieweit hier ein struktureller Unterschied

15 W. Hoffmann-Riem, Strukturen des Europäischen Verwaltungsrechts - Perspektiven der Systembildung, in: W. Hoffmann-Riem/E. Schmidt-Aßmann (Hrsg.), Strukturen des Europäischen Verwaltungsrechts, Baden-Baden 1999, S. 317 (317f.).

16 H. Peters, Die Verwaltung als eigenständige Staatsgewalt, Krefeld 1965; s. auch: W. Krebs, Verwaltungsorganisation, in: P. Kirchhof/ J. Isensee (Hrsg.), Handbuch des Staatsrechts V, 3. Aufl., Heidelberg 2005, $\mathbb{1} 108$ S. 457 (457ff.); ausf. H. Dreier/F. W. Graf/J. J. Hesse, Staatswissenschaften und Staatspraxis, Baden-Baden 2011, S. 182 ff.; sowie jetzt W. Hoffmann-Riem, Eigenständigkeit der Verwaltung, in: W. Hoffmann-Riem/E. Schmidt-Aßmann/A. Voßkuhle (Hrsg.), Grundlagen des Verwaltungsrechts, Bd. I, 2. Aufl., München 2012, $\mathbb{} 10$ Rn. 13, 16 ff., 56 ff., 70 ff.

17 Rossen-Stadtfeld, Medienaufsicht (Fn. 9), S. 462.

18 Rossen-Stadtfeld, Medienaufsicht (Fn. 9), S. $457 \mathrm{ff}$. 
zum traditionell der Rechtsfolgenseite zugeordneten Ermessen besteht, soll hier nicht thematisiert werden. ${ }^{19}$

Die „normative Ermächtigung“ der Verwaltung zur (partiellen) Letztentscheidung und damit die Anerkennung gerichtlicher Kontrollrestriktionen muss sich aus der Auslegung des Gesetzes ergeben. Die oben genannten funktionalen Argumente können noch keine Ermächtigung begründen, es muss sich zeigen lassen, dass der Gesetzgeber die Verwaltung ermächtigt hat. Die funktionsorientierte Ausgestaltung von Prozessen und Organisationen kann dabei aber Indizien liefern.

\section{Fallgruppen}

Es haben sich unterschiedliche Fallgruppen herausgebildet, die hier nur in Umrissen wiedergegeben werden können. ${ }^{20}$ Die folgende Darstellung orientiert sich an der von Rossen-Stadtfeld. ${ }^{21}$ Die Gründe, die eine normative Ermächtigung der Verwaltung in diesen Fällen der Rechtsprechung zufolge stützen, sind - wie gezeigt werden wird - vielfältig und $\mathrm{z}$. T. auch widersprüchlich.

Beurteilungsspielräume sind dort bejaht worden, wo die Anerkennung der Exekutive als funktionell eigenständige und eigenverantwortliche Staatsgewalt dies unabdingbar erscheinen ließ.22 Neben dem Politikvorbehalt zu Gunsten der Regierung und Ministerialbürokratie ist dies bei bestimmten Planungsentscheidungen, bei Entscheidungen mit planerischem Einschlag und bei solchen Entscheidungen angenommen worden, die wesentlich auf Prognosen beruhten. ${ }^{23}$ Die Aufgabe der (politischen) Gestaltung ist hier wesentlich der Verwaltung zugeordnet worden; strukturelle Voraussetzungen für eine Kompetenz der rechtsprechenden Gewalt sind für diese Aufgabe nicht erkennbar.

19 Zur Differenzierung zwischen Beurteilungsspielräumen und Ermessen: BVerwGE 72, $38(53)=$ NJW 1986, S. 796; M. Herdegen, Beurteilungsspielraum und Ermessen im strukturellen Vergleich, JZ 1991, S.747 (748).; K. Rennert, in: E. Eyermann/L. Fröhler (Begr.), VwGO Kommentar, 13.Aufl., München 2010, \114 Rn. 55; a.A. H.-J. Koch, Unbestimmte Rechtsbegriffe und Ermessensermächtigungen im Verwaltungsrecht, Frankfurt am Main 1979, S. 16 f.; zu den jeweils unterschiedlichen verfassungsrechtlichen Anforderungen siehe BVerwGE 79, 208 (213) = NJW 1988, S. 3221 (3221); vgl. auch die Verwischung der Trennlinie zwischen Ermessen und Beurteilungsspielräumen in BVerwGE 39, $197=$ NJW 1972, S. 158 (158); E 39, 355 = NJW 1972, S. 1411 (1411). Dazu W. Schmidt, Abschied vom "unbestimmten Rechtsbegriff ", NJW 1975, S. 1753 (1753); Vgl. zur historischen Entwicklung von der Lehre des Beurteilungsspielraums: Pache, Abwägung (Fn. 12), S. 56 ff.; M. Bullinger, Das Ermessen der öffentlichen Verwaltung, JZ 1984, S. 1001 (1003 ff.); vgl. Hoffmann-Riem (Fn. 16), \ 10 Rn. 71 und E. Schmidt-Aßmann, in: T. Maunz/G. Dürig (Begr.), Grundgesetz Kommentar, Lsbl. Stand: 60. Ergänzungslieferung 2010, Art. 19 Rn. 188 ff.

20 Die Bereiche anerkannter Beurteilungsspielräume zusammenfassend: S. Detterbeck, Allgemeines Verwaltungsrecht, 9.Aufl., München 2011, $\mathbb{8}$ Rn. 362; Pache, Abwägung (Fn. 12), S. 120 ff.; L. Jaeckel, Gefahrenabwehrrecht und Risikodogmatik: Moderne Technologien im Spiegel des Verwaltungsrechts, Tübingen 2010, S. 184 f, $201 \mathrm{f}$.

21 Rossen-Stadtfeld, Medienaufsicht (Fn. 9), S. 457.

22 BVerfGE 103, 141 (156f.) = NJW 2001, S. 1121 (1123).

23 BVerwGE 64, 238 (238 f.) = NJW 1982, S. 1168 (1169); BVerwG, DÖV 1979, S. 716 (716). 
Ein weiterer Typus von Beurteilungsspielräumen soll dort bestehen, wo die Verwaltung aus Sachgründen oder aufgrund von Funktionsgrenzen der Rechtsprechung typischerweise besser oder gar allein befähigt erscheint, die notwendigen Entscheidungen zu treffen. ${ }^{24}$ So begründete Beurteilungsspielräume sind angenommen worden für Prüfungsentscheidungen ${ }^{25}$ und prüfungsähnliche Entscheidungen ${ }^{26}$ sowie beamtenrechtliche Beurteilungen ${ }^{27}$ und Auswahlentscheidungen bei Bewerbungen. ${ }^{28} \mathrm{Zu}$ dem werden Spielräume für Handeln in gewissen von Rechtsprechung und Literatur als „komplex“ bezeichneten Problemlagen ${ }^{29}$ und für Entscheidungen, durch die in unübersichtlichen und schnell veränderlichen Regelungsbezügen dynamischer Grundrechtsschutz optimiert werden muss, ${ }^{30}$ anerkannt. Besondere Bedeutung ist in derartigen Fällen der Einmaligkeit und Unwiederholbarkeit der Entscheidungssituation $^{31}$ und dem Erfordernis fachlich spezialisierter Beurteilungs- und Entscheidungskompetenz zugemessen worden.

Ein dritter Typus von Beurteilungsspielräumen soll kollegialen Entscheidungsgremien einzuräumen sein, wenn solche Kollegialorgane weisungsunabhängig gestellt, staatsfern eingerichtet und fachkundig oder pluralistisch zusammengesetzt sind. ${ }^{32}$ Hier kann das Verfahren, in dem die Entscheidung der Verwaltung erarbeitet wird, durch die maßgeblichen gesetzlichen Konstituierungsregelungen so ausgestaltet sein, dass es auch die Funktionen eines vorverlagerten Rechtsschutzes miterfüllt. Zudem gibt es Fälle, bei denen das erkennbare Anliegen des Gesetzgebers im Vordergrund steht, für eine möglichst sachangemessene und effektive Bearbeitung besonderer

24 BVerfGE 84, 34 (49); E 88, 40 (56); E 103, 142 (156f.).

25 Seit BVerwGE 8, 272 = NJW 1959, S. 1842 (1842).

26 BVerwG, NVwZ 1991, S. 268; Schmidt-Aßmann (Fn. 19), Art. 19 Rn. 195 f.

27 BVerwGE 85, 177 (180); BVerwG, DVBl. 1984, S. 440 (440); H. Schulze-Fielitz, Neue Kriterien für die verwaltungsgerichtliche Kontrolldichte bei der Anwendung unbestimmter Rechtsbegriffe, JZ 1993, S. $772(776)$.

28 BVerwGE 115, 58 = NVwZ-RR 2002, S. 47 (47); OVG Lüneburg, IÖD 2008, S. 218 (218); OVG Lüneburg, NVwZ-RR 2003, S. 878 (878) = NordÖR 2003, S. 311 (311) m.w.N.

29 Zur Anerkennung eines besonderen pädagogischen Interesses für die Zulassung einer privaten Grundschule (Art. 7 Abs. 5 1. Fall GG) und den Maßstäben für ihre fachgerichtliche Überprüfung: BVerfGE 88, $40(58)=$ NVwZ 1993, S. 666 (666); BVerfGE 85, 36 (58) = NVwZ 1992, S. 179; Rennert (Fn. 19), $\mathbb{1 1 4}$ Rn. 71; Rossen-Stadtfeld, Medienaufsicht (Fn. 9), S. $463 \mathrm{f.}$

30 Z.B. im Atomrecht: BVerfGE 49, 89 (133) = NJW 1979, S. 359 (359); BVerwGE 72, 300 (316) = NVwZ 1986, S. 208 (208); E 78, 177 (180) = NVwZ 1988, S. 536 (536); VG Schleswig, NJW 1980, S. 1296 (1296); Rennert (Fn. 19), $\$ 114$ Rn. 72.

31 Die Unwiederholbarkeit und Einmaligkeit der Prüfungssituation kann allenfalls bei mündlichen, nicht aber bei schriftlichen Prüfungen für die Endgültigkeit der Bewertung durch den ursprünglichen Prüfer sprechen, vgl. F. Seebass, Die Prüfung - ein rechtsschutzloser Freiraum des Prüfers?, NVwZ 1985, S. $521(528)$.

$32 \mathrm{Zu}$ dem Gesichtspunkt besonderer Sachkunde innerhalb eines weisungsfreien, staatsfernen Kollegialorgans, s. jetzt BVerwG, NJW 2007, S. 2790 (2792 f.), mit der Entscheidung ist in entschiedener Abkehr von der früheren Rechtsprechung ein Beurteilungsspielraum der Weinprüfungskommission bejaht worden; Rennert (Fn. 19), $\mathbb{\$} 114$ Rn. 73 ff.; R. Stober, Gesetzesgebundenheit und Verwaltungsspielräume, in: Ders./W. Kluth (Hrsg.), Verwaltungsrecht I, 12. Aufl., München 2007, $\$ 31$ Rn. 25 f., m.w.N. 
Steuerungsprobleme Spielräume zu eröffnen, innerhalb derer staatliche Regulation durch gesellschaftlich-pluralistische oder fachlich-professionelle Selbstregulierung ersetzt wird.

Derzeit noch in der Diskussion ist eine Gruppe von Fällen, die sich im Bereich des Regulierungsverwaltungsrechts bewegen. Gegenstand der Diskussion sind zum einen der explizit vom Gesetzgeber eingeräumte Spielraum für die Bundesnetzagentur in $\$ 10$ Abs. 2 S. 2 TKG sowie der ebenfalls explizit benannte Spielraum der Einrichtungen der Selbstkontrolle gegenüber der Kommission für Jugendmedienschutz in $\$ 20$ Abs. 3 JMStV. ${ }^{33}$ Letztere werden hier nur insoweit einbezogen, als der Spielraum auf die Besonderheit von Entscheidungen im Bereich der Regulierung gestützt wird. Im Übrigen ist er ein Sonderfall, da es hier nicht um die Begrenzung verwaltungsgerichtlicher Kontrolle, sondern einer Kontrolle der Selbstkontrolle durch die Verwaltung geht.

\section{Vorgehen der Rechtsprechung am Beispiel des $\S 10$ TKG}

Eine Analyse der Rechtsprechung zeigt, dass ihr kein Konzept unterliegt, das auf einer Systematisierung beruht oder dass sie andersherum eine hilfreiche Basis wäre, um eine solche zu entwickeln. Zu beobachten ist eher ein eklektizistisches Vorgehen der Gerichte. ${ }^{34}$ Den Fallbeispielen der Rechtsprechung kommt in erster Linie heuristische Bedeutung zu. Alle Einzelfälle sind immer wieder im Rückgriff auf den rechtstheoretischen und funktionellrechtlichen Rahmen der normativen Ermächtigungslehre zu überprüfen. ${ }^{35}$

Die Marktregulierung im Telekommunikationsrecht ist insofern ein Sonderfall, als hier das europäische Recht eine Ermächtigung der nationalen Regierungsbehörden gebietet. ${ }^{36}$ Die Regelungen über das Marktdefinitions- und -analyseverfahren sind auf gemeinschaftsrechtliche Vorgaben zurückzuführen. Aus der Richtlinie 2009/140/ EG zur Änderung der Richtlinie 2002/21/EG über einen gemeinsamen Rechtsrahmen für elektronische Kommunikationsnetze und -dienste (Rahmenrichtlinie) ergeben sich zwar weitreichende Bestimmungskriterien, ${ }^{37}$ allerdings keine Vorgaben zur Aus-

33 K. Bosch, Die Kontrolldichte der gerichtlichen Überprüfung von Marktregulierungsentscheidungen der Bundesnetzagentur nach dem Telekommunikationsgesetz, Frankfurt am Main u.a. 2009, S. 47 ff.; Brunner, Beurteilungsspielräume im Jugendmedienschutzrecht (Fn. 9), S. 85 f.; Rossen-Stadtfeld, Medienaufsicht (Fn. 9), S. 457 ff.; hierzu allgemein: F. Schoch, Gerichtliche Verwaltungskontrollen, in: W, Hoffmann-Riem/E. Aßmann/A. Voßkuhle (Hrsg.), Grundlagen des Verwaltungsrechts, Bd. III, 2.Aufl., München 2012, $\$ 50$ Rn. 286 ff..

34 Rossen-Stadtfeld, Medienaufsicht (Fn. 9), S. 464; vgl. H. Faber, Verwaltungsrecht, 4. Aufl., Tübingen 1995, S. 103; K. Redeker, Von den Schwierigkeiten, ein Gesetz zu handhaben, NJW 1995, S. 2145 (2145 f.); mit Blick auf den besonders unsteten Gang der Rechtsprechung zu Beurteilungsspielräumen nach dem Gesetz über die Verbreitung jugendgefährdender Schriften vgl. Brunner, Beurteilungsspielräume im Jugendmedienschutz (Fn. 9), S. 71 ff.

35 Rossen-Stadtfeld, Medienaufsicht (Fn. 9), S. 464.

36 BVerwGE 131, 41 = NVwZ 2008, S. 1359, (1360).

37 Art. 7, 15, 16 Rahmenrichtlinie. 
gestaltung eines Beurteilungsspielraumes. Diese Entscheidung obliegt, sofern sich eine Richtlinie hierzu nicht äußert, den Mitgliedsstaaten. Der deutsche Gesetzgeber hat sich im $\$ 10$ Abs. 2 S. 2 TKG ausdrücklich für einen Beurteilungsspielraum entschieden, da es sich um eine Prognoseentscheidung mit weitreichenden Beteiligungsobliegenheiten handele. ${ }^{38}$ Das Bundesverwaltungsgericht legt dies weit aus ${ }^{39}$ und wird vom Bundesverfassungsgericht ${ }^{40}$ bestätigt. Für die verfassungsrechtliche Rechtfertigung vor allem am Maßstab des Art. 19 Abs. 4 GG reicht der Verweis auf das europäische Recht indes nicht. Die Ermächtigung bedarf eines Grundes, der die Verkürzung des Rechtsschutzes trägt.

So hat sich das Bundesverwaltungsgericht in einer Entscheidung mit dem Beurteilungsspielraum aus $\mathbb{1 0}$ Abs. 2 S. 2 TKG auseinander gesetzt, den es die Marktanalyse nach $\mathbb{\$} 11$ TKG ausdehnt. ${ }^{41} \mathrm{Um}$ diesen Spielraum zu begründen, greift das Bundesverwaltungsgericht auf zahlreiche an sich plausible Topoi der Beurteilungsspielraumlehre zurück, allerdings ohne sie in Beziehung zu setzen. ${ }^{42}$ In der Literatur wird zudem mit Recht angemerkt, dass das erste Urteil dazu eine differenzierte Analyse der zugrunde liegenden Entscheidungssituation vermissen lässt; ${ }^{43}$ neuere Entscheidungen sind nicht angetan, diese Kritik zu entkräften. ${ }^{44}$

Ein tragendes Argument des Bundesverwaltungsgerichts ist die besondere Struktur der Bundesnetzagentur, insbesondere ihre Entscheidungen in Kammern durch besonders sachkundige Expertinnen und Experten. ${ }^{45}$ Dieses Argument wird dahinge-

38 BT-DR 15/2316 v. 9.1.2004 S. 61.

39 BVerwG, NVwZ 2008, 575; BVerwGE 131, 41 = NVwZ 2008, S. 1359, (1359); BVerwG, ZUM-RD $2008,579$.

40 BVerfG, ZUM-RD 2012, 187.

41 BVerwG NVwZ 2009, S. 653 (653 ff.), im Anschluss an BVerwGE 131, 41 = NVwZ 2008, S. 1359 (1359) mit Anmerkungen von J. Oster, Marktdefinition und Marktanalyse im System des Rechtsschutzes, MMR 2009, S. 454 (454); J. Gräbig, Schwerpunkt 6: Domain- und Kennzeichenrecht, in: T. Hoeren/U. Müller (Hrsg.), Entwicklung des Internet- und Multimediarechts im Jahr 2008, MMR-Beil. 2009, S. 1 (25 f.); T. Attendorn, Das „Regulierungsermessen” - Ein deutscher "Sonderweg” bei der gerichtlichen Kontrolle tk-rechtlicher Regulierungsentscheidungen? MMR 2009, S. 238 (238); C. Franzius, Wer hat das letzte Wort im Telekommunikationsrecht?, DVBl. 2009, S. 409 (409); K. F. Gärditz, Regulierungsrechtliche Auskunftsanordnungen als Instrument der Wissensgenerierung, DVB1. 2009, S. 69 (69); H. Scherer, Die Entwicklung des Telekommunikationsrechts in den Jahren 2007 - 2009, NVwZ 2009, S. 1405 (1405). In neueren Entscheidungen übernimmt das BVerwG weitgehend die hier ausgearbeitete Argumentationsstruktur, vgl. BVerwG DÖV 2010, S. 1030 (1030) (zu Vorleistungsmärkten bei Mietleitungen) und BVerwG MMR 2009, S. 786 (787).

42 BVerwG NVwZ 2009, S. 653 (655), Anmerkung: T. Attendorn, Die Zieladäquanz der Regulierung: EG-rechtliche Impulse für eine wettbewerbsfördernde TK-Regulierung, NVwZ 2009, S. 19 (22) zu BVerwG NVwZ 2008, S. 575 (575).

43 Schoch (Fn. 33), $\$ 50$ Rn. 292 ff.; Attendorn, Tk-rechtliche Regulierungsentscheidungen (Fn. 36), S. 241; zustimmend U. Ellinghaus, Die Regulierungsverfügung in der verwaltungsgerichtlichen Praxis, CR 2009, S. 87 (89).

44 Vgl. etwa BVerwG, DöV 2010, S. 1030 (1030); BVerwG, DöV 2012, S. 325; BVerwG, NVwZ 2012, S. 694.

45 BVerwG, DVBl. 2008, S. 659 (662). 
hend kritisiert, ${ }^{46}$ dass damit jede Beschlusskammerentscheidung der Bundesnetzagentur mit der normativen Ermächtigung zur Letztentscheidung verbunden sein müsste, denn das Verfahren der Entscheidung im Rahmen der Marktanalyse unterscheidet sich nicht grundsätzlich von anderen, wenn es um die Organisation und Verfahren der Beschlusskammern geht.

Außerdem verweist das Bundesverwaltungsgericht auf den prognostischen Charakter der Entscheidungen der Bundesnetzagentur nach $\mathbb{S} \mathbb{\$} 10,11$ TKG. ${ }^{47}$ Auch diese Begründung ist so nicht hinreichend tragfähig. Denn es muss herausgearbeitet werden, wieweit sich dies im vorliegenden Fall signifikant von anderen prognostischen Entscheidungen unterscheidet, die die Verwaltung regelmäßig zu treffen hat. Zahlreichen ordnungsrechtlichen Verfügungen ist etwa regelhaft ein prognostisches Element im Hinblick auf die Entwicklung der Gefahr innewohnend, ohne dass die zuständige Behörde regelhaft ermächtigt würde, letztentscheidend tätig zu werden.

Schließlich wird, im Hinblick auf die konkreten Entscheidungen im Rahmen der Regulierungsverfügung gemäß $\$ 21$ TKG auf einen weiteren Typ der Beurteilungsspielraumslehre verwiesen, wenn das Bundesverwaltungsgericht die Vergleichbarkeit mit einem Planfeststellungsbeschluss bemerkt und dabei auf die „hohe Komplexität“ und „besondere Dynamik“ des Gegenstandsbereichs verweist. ${ }^{48}$ Bei diesen Passagen entsteht der Eindruck ein, der Begriff Komplexität mache die Schwierigkeit des Gerichts deutlich, eigene adäquate Beschreibungen anzufertigen. Vergleichbare Begründungsmuster finden sich bei Entscheidungen nach dem Gentechnikgesetz, wo ebenfalls recht pauschal auf „Komplexität“" verwiesen wird. ${ }^{49}$

Gerade im Bereich der Regulierungsverwaltung scheint es also einen Bedarf nach Beurteilungsspielräumen zu geben, der aber noch keine klare begriffliche Struktur und Grenze besitzt.

\section{Wissensverarbeitung und Entscheidungsqualität}

Die Wissensperspektive kann hier zur Schärfung der Dogmatik beitragen. Dass die entscheidende Ressource für staatliche Entscheidungen Informationen darstellen und, dass somit die Entscheidungsrichtigkeit in erheblichem Maße davon abhängt, wie das Informationsmanagement der Entscheidungsträger im Hinblick auf die in Rede stehende Entscheidung strukturiert ist, erscheint evident.

46 B. Kind, Zum Umfang der einem Netzbetreiber mit beträchtlicher Marktmacht durch die BNetzA auferlegbaren Verpflichtungen, CR 2008, S. 294 (295); Schoch (Fn. 33), \$ 50 Rn. 293.

47 BVerwGE 131, 41 (41) = NVwZ 2008, S. 1359 (1360) im Anschluss daran BVerwG, DöV 2010, S. 1030 (1030).

48 BVerwG ZUM-RD 2008, S. 579 (583); BVerwG DVBl. 2008, S. 659 (662).

49 BVerwG DÖV 2000, S. 37 (39). 
Aus dem Bereich der Informationstheorie haben bereits begriffliche Differenzierungen Eingang in die Rechtswissenschaft gefunden, die hier nur kurz in Erinnerung gerufen werden sollen, nämlich die zwischen Daten, Informationen, Wissen und Expertise. ${ }^{50}$ Mit dem Übergang von Daten - quasi dem Rohmaterial - zu Informationen ist bereits der Schritt zur Bedeutungsebene gemacht und dementsprechend hängt es von der zugrunde liegenden Gesellschafts-, Organisations- und Sprachtheorie ab, wie dieser Sinn theoretisch gefasst wird, jedenfalls wird er intern erst konstruiert. Vor diesem Hintergrund ist der in der systemtheoretisch inspirierten Literatur immer wieder zitierte Informationsbegriff von Bateson ${ }^{51} \mathrm{zu}$ verstehen, Information sei ein Unterschied, der (im verstehenden System) einen Unterschied macht.

Während Informationen systemspezifisch relevante Unterschiede bezeichnen, entsteht Wissen, wenn solche Informationen in bestimmte Erfahrungskontexte eingebunden sind und als bekannt vorausgesetzt werden können. Mit dem Begriff des Wissens wird auf die Ebene der Pragmatik Bezug genommen, Wissen ist also in systemisch relevante Praxis eingebaute Information. ${ }^{52}$ Damit ist auch klar, dass wir Wissen konstruieren und nicht in kleinen Päckchen - oder Akten - von A nach B tragen können. Von Expertise wird gesprochen, wenn Wissen strukturell auf konkrete Entscheidungssituationen beziehbar wird.

\section{Differenzierung von Wissen}

\section{Implizites und explizites Wissen}

Innerhalb des Wissens ist vor allem eine Unterscheidung rechtlich relevant: jene von implizitem und explizitem Wissen. Implizites Wissen oder stilles Wissen (vom englischen tacit knowledge abgeleitet) bedeutet, etwas zu können, ohne sagen zu können, wie“. Jemand „weiß, wie es geht“, aber sein Wissen steckt implizit in seinem Können, ihm fehlen die Worte, um dieses Können zu beschreiben oder es anderen verbal weiterzuvermitteln. Der Begriff wird auch in der Rechtswissenschaft gebraucht ${ }^{53}-$ die richtige Verortung bedarf jedoch eines weiteren Ausholens.

50 Instruktiv: H. Willke, Systemisches Wissensmanagement, 2. Aufl., Stuttgart 2001, S.1 ff.; zur rechtwissenschaftlichen Rezeption siehe: T. Vesting, Die Bedeutung von Information und Kommunikation für die verwaltungsrechtliche Systembildung, in: W. Hoffmann-Riem/E. Schmidt-Aßmann/A.Voßkuhle (Hrsg.), Grundlagen des Verwaltungsrechts, Bd. II, 2.Aufl., München 2012, \$ 20 Rn. 27.

51 Information ist nicht eine Differenz in der Außenwelt, die von einem System aufgenommen wird, ohne sich dabei zu verändern, sondern sie ist eine Differenz, die wiederum eine Differenz in diesem System erzeugt. Nach der Definition von G. Bateson, Steps to an ecology of mind, Chicago u.a., 5.Aufl., University of Chicago Press 2000, S. 453 (453), Zitat nach Qvortrup, The Social Significance of Telematics, 1993, S. 10 (10); dt. 1985, S. 582 (582).

52 H. Willke, Dimensionen des Wissensmanagements - Zum Zusammenhang von gesellschaftlicher und organisationaler Wissensbasierung, In: G. Schreyögg/P. Conrad (Hrsg.), Jahrbuch für Managementforschung VI, Wiesbaden 1996, S. 191.

53 H. Burkert/M. J. Eppler, Wissensmanagement im Recht - Möglichkeiten und Grenzen einer wissensorientierten Rechtsbetrachtung, MMR 1999, S. 627 (627). 
Der Begriff des impliziten Wissens geht auf den ungarisch-englischen Naturwissenschaftler und Philosophen Michael Polanyi zurück. Besser als in der deutschen Übersetzung (,implizites Wissen“) wird in dem von ihm verwendeten Begriff „tacit knowing " deutlich, dass das Interesse nicht primär dem Wissen, sondern vielmehr dem Können gilt - nicht kognitiven Strukturen also, sondern mentalen Prozessen. ${ }^{54}$ Der Blick richtet sich auf Wahrnehmungs-, Entscheidungs- und Handlungsdispositionen und die ihnen entsprechenden Formen der mehr oder weniger intuitiven Performanzregulation.

Nach Neuweg lassen sich vier Formen des Konzeptes „implizites Wissen“ unterscheiden: ${ }^{55}$

1. „in actu implizit“ („intuitiv“): Eine Person handelt kompetent, erinnert während des Handelns aber keine Handlungsregeln, sondern agiert ,automatisch “, „,spontan“ oder ,intuitiv“.

2. „nicht verbalisierbar“: Eine Person handelt kompetent, erinnert während des Handelns aber keine Handlungsregeln, sondern agiert - wie in Fall 1 - ,automatisch“, „spontan“ oder „intuitiv“. Auch im Nachhinein kann sie auf Anfrage keine solchen Regeln benennen. Ein Beobachter könnte dies aber.

3. „nicht formalisierbar“: Eine Person handelt kompetent, erinnert während des Handelns aber keine Handlungsregeln, sondern agiert ,automatisch“, „spontan“ oder „intuitiv“. Auch im Nachhinein kann sie auf Anfrage keine solchen Regeln benennen. Das gilt aber nicht nur für den Handelnden, sondern auch für Beobachter, die versuchen, das fragliche Können über Regeln zu beschreiben.

4. „erfahrungsgebunden“: Damit ist ein Wissen gemeint, das sprachlich nicht oder kaum weitergegeben werden kann. In solchen Fällen muss der Betreffende durch eigene Erfahrung oder am Modell lernen, das ihm vorzeigt, was nicht vorgesagt werden kann.

Empirisch wird implizites Wissen in der Regel als Differenzgröße zwischen Können und explizitem Wissen aufgefasst und auch entsprechend gemessen. Es wird einerseits erfasst, was eine Person kann, und andererseits gemessen, was sie berichtbar weiß; gleichsam als Differenz ergibt sich dann, was (nur) implizit „gewusst“ wird.

Wissen können nicht nur Menschen, sondern auch Organisationen. Wiegand definiert Organisationen sogar in Abhängigkeit vom Wissen: ${ }^{56}$

54 G. H. Neuweg, Das Schweigen der Könner, Linz 2006, sowie Könnerschaft und implizites Wissen, 2. Aufl., Münster u.a. 2006.

55 G. H. Neuweg, Impliziertes Wissen als Forschungsgegenstand, in: F. Rauner (Hrsg.), Handbuch Berufsbildungsforschung, 2. Aufl., Bielefeld 2005, S. $581 \mathrm{ff}$.

56 H. Wiegand, Die lexikographische Definition im allgemeinen einsprachigen Wörterbuch, in: F. J. Hausmann/O. Reichmann/H. Wiegand/L. Zgusta, Wörterbücher I, 1991, S. 530 (530ff.). 
„Die Organisation wird als offenes soziales bzw. soziotechnisches System mit angebbarem Mitgliederkreis verstanden, das zeitpunktbezogen über verschiedene Arten und Formen von Wissen verfügt bzw. gespeichert hat, die lernebenenspezifisch und zustandsgebunden generiert wurden."

Die Organisation wird mit anderen Worten als „Wissenspeicher“ konzipiert. Entlang dieses (organisationsspezifischen) Wissens, das der Organisation ein bestimmtes Verhaltenspotential eröffnet, ist die Organisation von der Umwelt und von anderen Organisationen sowohl aus einer Außen- als auch aus einer Innenperspektive abgrenzbar.

Ein Thema in der Organisationsforschung ist, wie implizites Wissen der Mitglieder der Organisation in explizites verwandelt werden kann, um es von der Person abzulösen. ${ }^{57}$ Aber Organisationen können auch selbst implizites Wissen haben. Im vorliegenden Kontext ist interessant, inwieweit es auch bei ihnen Grenzen der Explizierbarkeit von Wissen gibt. Jedenfalls die Typen 3 und 4 schienen auf Organisationen übertragbar. Es gibt Grenzen der Formalisierbarkeit von Wissen, Grenzen dessen, was sich aktenmäßig erfassen lässt. Auch ist es denkbar, dass sich soziale Praxen bilden, an die das Wissen so geknüpft ist, dass es als implizit erscheint. Darauf wird am Ende anhand von Beispielen aus der Regulierungsverwaltung zurück zu kommen sein.

\section{Wissen und Nichtwissen}

Auch beim Nicht-Wissen lassen sich für das Recht relevante Differenzierungen ausmachen. So bei der Frage des Wissens um das Nichtwissen. Hier ist explizit bewusstes Nichtwissen von (vollständig) unerkanntem Nichtwissen zu unterscheiden. Man kann in der Folge auch von spezifischem und unspezifischem Nichtwissen sprechen. ${ }^{58}$

Nichtwissen erscheint keineswegs als negative Seite des Wissens und lediglich als Feind eines Rationalitätsversprechens des modernen Staates. Nichtwissen ist zum Teil nicht hintergehbar, zum Teil aber bewusst gewolltes Nichtwissen - bei sich selbst etwa, um sich zu exkulpieren, oder bei anderen, um Machtspielräume zu kontrollieren.

Die zeitliche Stabilität und Dauerhaftigkeit von Nichtwissen ist wiederum eine Klassische Differenzierung, also ob bloß temporäres Nichtwissen (Noch-Nicht-Wissen) oder unauflösbares Nichtwissen (Nicht-Wissen-Können) vorliegt.

57 Katenkamp, Implizites Wissen in Organisationen, Wiesbaden 2011.

58 W. Hoffmann-Riem, Wissen als Risiko - Unwissen als Chance, in: I. Augsberg (Hrsg.), Ungewissheit als Chance, Tübingen 2009, S. 17 (27). 


\section{Verwaltungsentscheidungen und gerichtliche Kontrolle in der Wissensperspektive}

Aus einer informationstheoretischen Perspektive lässt sich die Frage nach Beurteilungsspielräumen dahingehend reformulieren, dass nach besonderen Entscheidungstypen und der dafür adäquaten Informationsverarbeitung gefragt wird. Es kann im Hinblick auf die zu lösende Problemlage die Informationsverarbeitung der Verwaltung so gestaltet sein, dass dem Gesichtspunkt der Entscheidungsrichtigkeit nur hinreichend Rechnung getragen wird, indem deren normative Ermächtigung zur Letztentscheidung erfolgt und dies als Kehrseite zu einer eingeschränkten Kontrolldichte der Gerichte führen muss.

Skizziert man grob die Entscheidungssituation des Gerichts, so ist sie durch Merkmale geprägt, die vor allem eine Reliabilität der Entscheidung sicher stellen sollen (jedes Gericht würde grundsätzlich zu dem gleichen Schluss kommen), es gibt keine (Einzelrichter) oder eingeschränkte (Kollegial-Gerichte) Perspektivenvielfalt bei der Informationsverarbeitung und einem systematisch eingeschränkten Möglichkeitsraum für die „Zufuhr“ von Informationen; ${ }^{59}$ gerichtlich geprüft werden kann, was auf diesen Kanälen an expliziertem Wissen zur Verfügung steht. Zu letzteren zählt auch der Sachverständige, so dass zu Recht darauf hingewiesen wird, dass die vom Bundesverwaltungsgericht auch in der oben genannten Entscheidung zum Telekommunikationsrecht angeführte größere „Sachnähe“ der Verwaltung allein zur Begründung von Beurteilungsspielräumen nicht ausreichen kann; sie ist nur dann ein Argument, wenn die zu einer sachgerechten Entscheidung erforderlichen Informationen durch Sachverständige nicht beigebracht werden können, das Gericht also auch durch Sachverständige das erforderliche Entscheidungswissen nicht erlangt.

Entscheidungsqualität und Legitimation. Bislang wurde eine Dimension noch nicht beleuchtet, die bei der Zuweisung von Letztentscheidungskompetenz eine Rolle spielt, nämlich den Legitimationszusammenhang. ${ }^{60}$ Bei den politischen oder politiknahen Entscheidungen geht es nicht primär darum, die optimale Entscheidung zu treffen, sondern - das ist das Wesen der Politik - Kontingenz durch eigene willkürliche aber unmittelbar zu legitimierende Entscheidung zu reduzierten. Insofern liegt in diesem Typus ein Sonderfall. Verallgemeinerbar ist lediglich, dass (alternative) Legitimationsmechanismen auch bei anderen Entscheidungen beachtlich sind und neben der hier im Mittelpunkt stehenden Frage der prozeduralen und organisatori-

59 Problemverschärfend tritt hinzu, dass diese Entscheidungsmaßstäbe längst nicht mehr einem Pool von Moralsystemen, Wert- ordnungen oder Konventionen entnommen werden können, die zwar außerrechtlich bleiben, aber doch wenigstens von jeweils maßgebenden gesellschaftlichen Eliten als verbindlich anerkannt werden. Das ist schon früh festgestellt worden, s. etwa F. Werner, Das Problem des Richterstaats, in: K.A. Betterman/C. H. Ule (Hrsg.), Recht und Gericht in unserer Zeit. Reden, Vorträge, Aufsätze 1948 - 1969, S. 176 (189); E. Forsthoff, Lehrbuch des Verwaltungsrechts I, 10. Aufl., München u.a. 1973, S. 68 (68); H. Rossen-Stadtfeld, Gesetzesvollzug durch Verhandlung Kann der Verwaltungsrichter von der Verwaltung lernen?, NVwZ 2001, S.361 (366).

60 H.-H. Trute, Die demokratische Legitimation der Verwaltung, in: Hoffmann-Riem et al. (Hrsg.), Grundlagen d. Verwaltungsrechts (Fn. 16), Bd. I, $\$ 6$ Rn. 14, 56. 
schen Ausstattung der Verwaltung zur Sicherstellung möglichst richtiger Entscheidungen auch ihre spezifische Ausstattung mit Legitimationskapital in Rechnung zu stellen ist. Dies bleibt im Folgenden ausgeblendet, hat aber gerade bei der Fallgruppe unabhängiger Gremien eine Bedeutung.

\section{Zu den informationalen Strukturmerkmalen von Entscheidungen}

Qualität von Entscheidungen hängt von verfügbarem Entscheidungswissen ab. Dabei ist das Wissen unvollständig, keineswegs nur, aber natürlich in besonderer Weise im Bereich prognostischer Entscheidungen. Es sollte aber klar sein, dass sich keine Entscheidung auf vollständige Informationen stützen kann; Entscheidung unter Nichtwissen ist die Regel, nicht die Ausnahme. Nicht nur die Kontingenz, die eine Entscheidung zur Entscheidung macht, beruht darauf, sondern wie Lubmann betont, auch der Spaß am Entscheiden. ${ }^{61}$

Es gibt allerdings Differenzierungen. Im Zustand der Entropie, in dem keine entscheidungsrelevante Information zur Verfügung steht, sind alle Deutungsvarianten gleich gut und folglich die Entscheidung vollständig kontingent. Die Informationstheorie hat oberhalb dessen unterschiedliche informationale Strukturmerkmale bis hin zu Defekten von Entscheidungen ausgemacht. Sie werden im Folgenden aufgegriffen, um im Folgenden einige Typen von Entscheidungen zu formen, bei denen sich Verwaltung und Gericht organisatorisch im Hinblick auf das erforderliche Wissensmanagement unterscheiden. Sie ist nicht vollständig; es erschließt sich unmittelbar, dass etwa auch Prognoseentscheidungen eine spezifische Wissensdimension haben, die mit der Frage des Umgangs mit Nicht-Wissen verbunden ist; dies bedarf einer eigenständigen Analyse.

\section{Typ 1: Unhintergehbare Flüchtigkeit von Wissen}

Strukturelle Defizite kann es zum einen geben, wenn Informationen flüchtig sind. Zur Entscheidungsfindung im Hinblick auf die Leistungen eines Prüflings in einer mündlichen Prüfung gehören neben dem reinen Inhalt der Antworten auch Faktoren wie etwa das Reaktionstempo, Responsivität und Interaktion mit den anderen Prüflingen, wenn es sich um eine Gruppenprüfung handelt. Damit sind Kriterien beschrieben, die sich nicht oder nur mit unverhältnismäßigem Aufwand in Prüfungsprotokollen niederlegen und dem Gericht zur Verfügung stellen lassen. Die Ungleichheit der Entscheidungssituation schlägt sich dann als Beeinträchtigung der Chancengerechtigkeit der Prüflinge nieder, wenn bei einigen die Note vom Gericht festgestellt würde. Daher nimmt die herrschende Meinung zu Recht an, dass die Bewertung von Prüfungsleistungen insoweit nur eingeschränkt gerichtlich kontrollierbar ist. Bei der Körungsprüfung hat das Bundesverwaltungsgericht die Unwiederholbarkeit sogar explizit als hinreichende Bedingung für einen Beurteilungsspielraum

61 N. Luhmann, Organisation und Entscheidung, 2. Aufl., Wiesbaden 2006, 132 ff. 
genannt. ${ }^{62}$ Wo der Gesetzgeber eine Entscheidung im Gesamteindruck der mündlichen Prüfung verlangt, spricht er die Ermächtigung aus. Das Argument gilt allerdings nur für mündliche Prüfungen und nicht etwa für schriftliche und auch nicht für Beamtenleistungsbewertungen. ${ }^{63}$

Prinzipiell könnte der Gesetzgeber dem Problem der Flüchtigkeit auch durch spezifische Dokumentationspflichten begegnen, dies stößt allerdings schnell an funktionale Grenzen. ${ }^{64}$ Insoweit bleibt die Flüchtigkeit unhintergehbar.

Ein auf den ersten Blick ebenso instruktives Beispiel für Flüchtigkeit ist die Frage, die das Bundesverwaltungsgericht 2007 zu entscheiden hatte. ${ }^{65}$ Es ging um die Beurteilung der zuständigen Behörde, ob ein Wein in Aussehen, Geruch und Geschmack frei von Fehlern ist und so die Voraussetzung zur Erteilung einer amtlichen Prüfnummer vorliegt ( $\$ 19$ WeinG, $\mathbb{S} 21,22,24,25 \mathrm{WeinV}$ ). Das Gericht hat unter ausdrücklicher Aufgabe seiner alten Rechtsprechung ${ }^{66}$ einen Beurteilungsspielraum anerkannt.

Hier ist aufgrund des Zeitfortschritts das Rohmaterial von Wissen für den Richter nicht verfügbar. Der Wein zum Zeitpunkt der sensorischen Prüfung der Behörde existiert nicht mehr; er ist gealtert und hat sein Aussehen, Geruch und Geschmack also gerade die zu prüfenden Eigenschaften - geändert. Dieses Problem kann die Rechtsordnung umgehen, indem sie für die Entscheidung auf den Zeitpunkt der mündlichen Verhandlung abstellt. Dies trägt aber, wie der Fall zeigt, nur in typischen Fallkonstellationen. Das Berufungsgericht hatte den Sachantrag des Klägers dahin verstanden, dieser begehre die Feststellung, dass die Versagung der amtlichen Prüfungsnummer im Zeitpunkt des Widerspruchsbescheides rechtswidrig gewesen sei. Bei dieser Konstellation wäre der Fall flüchtigen Wissens gegeben.

Dieser Fall führt aber über den ersten Typus der Flüchtigkeit von Wissen hinaus. Geht man davon aus, dass dem Richter das Datum - also der Wein - zur Verfügung steht, weil es auf den Zeitpunkt der mündlichen Verhandlung ankommt, ist die Fra-

62 BVerfG NVwZ 1981, S. 568 (568).

63 Die Unwiederholbarkeit und Einmaligkeit der Prüfungssituation kann allenfalls bei mündlichen, nicht aber bei schriftlichen Prüfungen für die Endgültigkeit der Bewertung durch den ursprünglichen Prüfer sprechen: F. Seebass, Rechtsschutzloser Freiraum (Fn. 31), S. 528 f.; P. Gubl, Prüfungen im Rechtsstaat, Bad Honnef 1978, S. 37; B.O. Bryde, Die Kontrolle von Schulnoten in verwaltungsrechtlicher Dogmatik und Praxis, DÖV 1981, S. 193 (198f.); W. Grupp, Gerichtliche Kontrolle von Prüfungsnoten, JuS 1983, S. 351 (355); P. Theuersbacher, Die gerichtliche Kontrolle im Multiple-Choice-Verfahren der staatlichen, ärztlichen und pharmazeutischen Prüfungen, BayVBl 1984, S. 129 (131). Siehe auch zur Entwicklung der Rechtsprechung im Prüfungsrecht: E. Pache, Abwägung (Fn. 12),S. 128 ff.; zu beamtenrechtlichen Leistungsbewertungen BVerwGE 21, 127 (129f.); E 60, 245; restriktiver BVerwGE 106, 263 (266 f.); Vgl. auch BVerfG NVwZ 2002, S. 1368 (1368); zu Prüfungsentscheidungen: BVerfGE 84, 34; BVerwGE 91, 262; E 92, 132; E 99, 185; E 104, 203.

64 An Beispiel des Schulrechtes J. Rux, Die pädagogische Freiheit des Lehrers, 2002, Berlin, S. 66 f. und S. 70.

65 BVerwGE 129, 27 = NJW 2007, S. 2790 (2790 f.), Entscheidungsbesprechung V. Schoene, FD-GewRS 2007, 239249.

66 BVerwGE 94, 307 = NVwZ 1995, S. 707 (709). 
ge, ob es auf der Ebene von Wissen oder Expertise strukturelle Defizite gibt. Das Bundesverwaltungsgericht hat sich in der Begründung des Spielraums wie üblich auf einen Sammlung von Gründen berufen und den Typus einer Entscheidung angenommen, dem hohem Maße wertende Elemente anhaften und für den das Gesetz deshalb ein besonderes Verwaltungsorgan für zuständig erklärt, das weisungsfrei, mit besonderer fachlicher Legitimation und in einem besonderen Verfahren entscheidet; dies zumal dann, wenn es sich um ein Kollegialorgan handelt, das mögliche Auffassungsunterschiede bereits in sich zum Ausgleich bringt und die zu treffende Entscheidung damit zugleich versachlicht. Damit ist die Notwendigkeit diskursiver Produktion von Entscheidungswissen und damit ein weiterer Entscheidungstypus angesprochen.

\section{Typ 2: Notwendigkeit diskursiver Produktion von Entscheidungswissen}

Auch der traditionelle Typus von Beurteilungsspielräumen auf der Ebene des Wissens, der Entscheidungen von in besonderer Weise zusammengesetzten Gremien zum Inhalt hat, lässt sich so einordnen. Zum einen sind hier häufig neben Wissensfragen - etwa der Sachverhaltsermittlung und Bewertung und der Prognose - auch Wertungsspielräume ${ }^{67}$ präsent, die etwa die Konkretisierung der Ziele der Regelung betreffen. ${ }^{68}$ Zum anderen aber werden derartige Gremien zur Entscheidung eingesetzt, weil der Gesetzgeber der Auffassung ist, dass zur sachgerechten Entscheidung die Informationen diskursiv verarbeitet werden müssen, um das nötige Entscheidungswissen zu erzeugen. Die Informationsforschung bestätigt, dass die Konstruktion von Wissen beim Problemlösen in Gremien eine anerkannte Sonderform darstellt. ${ }^{69}$ Der Wert besteht darin, dass im Diskurs unterschiedliche Wissenshorizonte und Deutungsmuster unmittelbar interagieren und so eine Ebene von Handlungswissen erreicht wird, die ein Einzelentscheider nicht erreicht. Für diesen Mehrwert reicht grundsätzlich der Dialog und eine gewisse Perspektivenvielfalt der Mitglieder; die anderen in der Rechtsprechung genannten Merkmale wie Weisungsunabhängigkeit $^{70}$ haben also mit diesem Kriterium der Entscheidungsqualität nicht unmittelbar zu tun und müssen sich anders begründen, etwa durch Absicherung der spezifischen Entscheidungsform gegen Intervention.

Im Hinblick auf derartige Gremienentscheidungen ist also das Verfahren und die Zusammensetzung des Gremiums daraufhin zu untersuchen, ob sie eine diskursive Wissensverarbeitung beinhalten, die von Verwaltungsgerichten in dieser Weise nicht substituiert werden kann, auch nicht durch die Befragung von Sachverständigen un-

67 Bewertungsspielräume bleiben in diesem Beitrag im Übrigen unberücksichtigt, vgl. aktuell BVerwG NVwZ-RR 2011, S. 279 (281).

68 Bsp. Jugendschutz.

69 Siehe V. Born, Problemorientiertes Wissensmanagement in der Automobilindustrie: Ein interdisziplinärer Ansatz, Wiesbaden 2008, S. 35 ff.

70 BVerfGE 3, 377 (381) = NJW 54, S. 833 (833); BVerfG NJW 1969, S. 2192 (2193). 
terschiedlicher Disziplinen. Bei der Kommission Jugendmedienschutz und der Bundesprüfstelle für Jugendgefährdende Medien ist dies der Fall, ${ }^{71}$ nicht aber bei der Bundesnetzagentur, deren Beschlusskammern nicht nach Gesichtspunkten der Perspektivenpluralität zusammengesetzt sind. ${ }^{72}$

Dass aber - um beim Beispiel Telekommunikation zu bleiben - der Bundesnetzagentur die Aufgabe zukommt, die Stellungnahmen der anderen Regulierungsbehörden in die eigene Erkenntnisgewinnung zu integrieren, enthält ein Moment der Wissensproduktion, das der in Gremien strukturähnlich ist. Die Marktfestlegung vollzieht sich - jedenfalls dem gesetzlichen Programm nach - in einem Prozess, der wechselseitig transnationale Einflüsse einbezieht und so eine Perspektivenvielfalt integriert.

\section{Typ 3: Die Notwendigkeit impliziten Wissens}

Jede Behörde greift bei Entscheidungen auch auf implizites Wissen zurück, ungeachtet des Prinzips der Aktenführung in $\$ 29 \mathrm{VwVfG}$, das auf Formalisierung und Explizierung des Wissens zielt. Die Frage ist, ob es bestimmte Formen der Verwaltung gibt, bei denen implizites Wissen strukturell eine so entscheidende Rolle spielt, dass ohne dieses die Entscheidungsqualität signifikant reduziert wird, und der Gesetzgeber die Verwaltungsverfahren daher entsprechend gestaltet hat.

Schon in die Richtung einer Begründung für Beurteilungsspielräume im Rahmen von Entscheidungen regulierender Verwaltung weist - allerdings nur für den Jugendmedienschutz - Helge Rossen-Stadtfeld. ${ }^{73} \mathrm{Er}$ sieht gerade in komplexen Konfigurationen der Selbst- und Ko-Regulierung Unbestimmtheit von Rechtsbegriffen als Steuerungsmedium. Für die Begründung eines Beurteilungsspielraums stützt er sich aber letztlich wieder auf die traditionellen Topoi sachverständige Kollegialität, Gruppenpluralität und Staatsferne des Gremiums.

An dieser Stelle ist festzuhalten: Es scheint eine möglicherweise neue Typenbildung im Bereich der Beurteilungsspielraumslehre erforderlich zu sein, die im Bereich der Regulierungsverwaltung angesiedelt ist, deren Begründung allerdings noch an Schärfe mangelt und die bislang überwiegend durch kumulativen Rückgriff auf die Argumente, die die anderen Typen stützen, begründet wird.

Zunächst ist zu klären, was die Besonderheit der Handlungsform „Regulierung“ ausmacht. In Anlehnung an Eifert, ${ }^{74}$ der wiederum politikwissenschaftliche Bestim-

71 Daher zur KJM zu Recht Rossen-Stadtfeld, Medienaufsicht (Fn. 9), S. 474; a.A. VG Berlin mit Anmerkung von Liesching, ZUM 2006, S. 779 (784). Einen Beurteilungsspielraum der KJM ablehnend: Brunner, Beurteilungsspielräume im Jugendmedienschutz (Fn. 9), S. 182 f.

72 Bosch, Die Kontrolldichte der gerichtlichen Überprüfung von Marktregulierungsentscheidungen, 2009, S. 74ff.; aA Liebschwager, Gerichtliche Kontrolle administrativer Regulierungsentscheidungen, 2005, S. 165 f., der in den Beschlusskammerverfahren einen vorweggenommenen Rechtschutz erkennt.

73 Rossen-Stadtfeld, Medienaufsicht (Fn. 9), S. 457 ff.

74 M. Eifert, Regulierungsstrategien, in: Hoffmann-Riem et al. (Hrsg.), Grundlagen d. Verwaltungsrechts (Fn. 16), Bd. I, $\mathbb{S} 19$ Rn. 1 ff. 
mungen aufgreift, kann Regulierung in einem weiten Verständnis begriffen werden als gewollte staatliche Beeinflussung von Prozessen, die einem spezifischen, über den Einzelfall hinaus gehenden Ordnungszweck gehorcht und Recht als Medium und Grenze hat. Zentral ist also, dass Entscheidungen auf einen Prozess zielen und über den Einzelfall hinaus programmiert werden.

Der Bereich der Regulierungsentscheidungen verweist auf Besonderheiten auf einer noch höheren Stufe der Informationsverarbeitung, nämlich der Expertise. Wird ein sozialer Prozess durch einen speziell dafür eingerichteten „Regulator" 75 beeinflusst, so wird damit auch eine bestimmte Form der Wissensverarbeitung ermöglicht. Die Herausbildung besonderer Regulierungsinstanzen geht einher mit einer Lernfähigkeit $^{76}$ der entsprechenden Institutionen, so dass in Entscheidungen auch systematisch implizites Wissen eingeht. Auch Gerichte verfügen über selbst konstruiertes implizites Wissen, sie können sich aber das der Verwaltung nicht „zuführen“, dies liegt wie gesehen im Wesen des impliziten Wissens. Ihre Begründungskultur ist zudem auf die Prüfung expliziten Wissens bezogen. Wo also implizites Wissen entscheidungserheblich wird, ergeben sich Kontrollschwächen.

Dass dieses Problem gerade bei Regulierungsverwaltung auftritt, hat mehrere Gründe. Regulierungsentscheidungen sind rekursiv miteinander verknüpft. Plastisch wird dieser Prozess bei dem alten Konzept der Responsive Regulation von Ayres/ Braithwaite. ${ }^{77}$ Sie fassen Regulierung als eine Art „Spiel“ zwischen Reguliertem und Regulierer auf, bei dem eine Entscheidung der Regulierungsbehörde im Kontext quasi als ein Zug im Spiel zu bewerten ist, so dass sich daraus Restriktionen für die Möglichkeiten von Gerichten ergeben, die Entscheidung zu substituieren. ${ }^{78}$ Auch wenn dieses Konzept moderne Regulierung nur in einem unvollkommenen spieltheoretischen Modell erfasst, erscheint die rekursive Verschleifung auch bei anderer theoretischer Modellierung als Charakteristikum von Regulierung. Wissen - und auch gerade Nichtwissen, etwa Regulierung durch Management von Wissensvorsprüngen - kann hier eine zentrale Ressource sein.

Der Regulierer ist Teil des sozialen Prozesses, den er reguliert. Dies ist der Kopplung an soziale Praxen, die die vierte der oben eingeführten Formen des impliziten Wissens darstellt, jedenfalls strukturähnlich. Mit dieser Rolle geht nämlich auch einher, dass das Wissen um die Folgen einer Entscheidung für andere Entscheidungen bedeutsam

75 A. Baudrier, Independent Regulation and Telecommunications Performance in Developing Countries, zu finden unter: http:/citeseerx.ist.psu.edu/viewdoc/download?doi=10.1.1.202.4868\&rep=rep1\&type=pdf.; grundlegend zu Regulierungsbehörden: R. Baldwin/M. Cave, Understanding Regulation: Theory, Strategy and Practice, Oxford u.a., Oxford University Press 1999.

76 Eifert (Fn. 66), $\mathbb{1} 19$ Rn. $1 \mathrm{ff}$.

77 I. Ayres/J. Braithwaite, Responsive regulation: transcending the deregulation debate, New York u.a., Oxford University Press1992.

78 ebenso Rossen-Stadtfeld, Medienaufsicht (Fn. 9), S. 467. 
wird. Dies mag das Bundesverwaltungsgericht im Sinn gehabt haben, als es recht pauschal auf die besondere Komplexität der Entscheidungen im Bereich der Marktregulierung nach $\mathbb{S} \$ 10 \mathrm{ff}$. TKG verwiesen hat.

Typisch für Regulierungsverwaltung ist daher auch eine komplexe Wissensinfrastruktur, die das eigene explizite und implizite Wissen ständig mit Expertise in Form von Gutachten, sachverständigen Beiräten und Anhörungen kontrastiert und ergänzt. Dazu gehört auch, dass dezentrales Wissen etwa der Verbände und Unternehmen der betreffenden Branchen verfügbar gemacht wird, aber auch darüber hinaus von NGOs oder anderen Wissensträgern. ${ }^{79}$ Die Regulierungsbehörde kann organisatorisch und prozedural gerade darauf programmiert sein, dass jeweils nötige Entscheidungswissens in diesem Prozess jeweils zeitbezogen zu konstruieren.

Dort, wo Regulierungsentscheidungen auf der so erzeugten impliziten Expertise beruhen, ist sie auch durch einen durch Sachverständige beratene Richter nicht substituierbar. Und damit nicht genug: Das Wissen um die materielle Angreifbarkeit der Regulierungsentscheidung schwächt die Handlungsmöglichkeiten des Regulierers und eine Entscheidung eines Gerichtes kann die Statik des Regulierungssystems verändern, ohne diese Folge im Entscheiden reflektieren zu können. Plakativ gesprochen, regulieren die Gerichte gezwungenermaßen mit; dies mag ihre in einigen Entscheidungen zwischen den Zeilen lesbare Reaktanz erklären.

Freilich muss sich im Einzelfall begründen lassen, dass die nur begrenzt gerichtlich kontrollierte Ausfüllung des unbestimmten Rechtsbegriffs gerade mit den eben ausgeführten Regulierungsfunktionen verbunden ist und der Gesetzgeber der Behörde mit Blick darauf die Deutungshoheit zuerkannt hat. Nimmt man sich die Entscheidung der BNA nach $\mathbb{1} 10$ TKG vor, so ist die Einbindung in das Konzept der Regulierung evident und zwar auch in der Breite, wie sie das Bundesverwaltungsgericht angenommen hat. Entscheidungen der Marktregulierung müssen nach Art. 8 Abs. 4 der Zugangsrichtlinie eine problemadäquate Maßnahme darstellen, mit der Folge, dass eine auf die effektive und dauerhafte Beseitigung von Wettbewerbsschranken zielende Lösung zu finden ist. ${ }^{80}$ Die Einzelentscheidung ist also immer mit ihren Folgen für das Gesamtsystem zu betrachten, eine typische Regulierungskonstellation. Entsprechend sind die Wissensstrukturen - Beirat ( $\$ 120$ TKG), Berichtswesen ( $\mathbb{S} 121 \mathrm{f}$. TKG), wissenschaftliche Beratung ( $\$ 125 \mathrm{TKG})$ - ausgerichtet, die eine permanente Marktbeobachtung ermöglichen.

79 Vgl. T. Vesting, Satzungsbefugnis von Landesmedienanstalten und die Umstellung der verwaltungsrechtlichen Systembildung

auf ein "Informationsverwaltungsrecht“, Die Verwaltung, 2002, S. 433, (451 ff.); siehe auch T. Vesting, Die Bedeutung von Information und Kommunikation für die verwaltungsrechtliche Systembildung, in: Hoffmann-Riem et al. (Hrsg.), Grundlagen d. Verwaltungsrechts (Fn. 45), Bd. II, $\$ 20$ Rn. $41 \mathrm{ff}$.

80 EuGH MMR 2009, S. 95 (95); EuGH NVwZ 2010, S. 370 (370); B. Holznagel/A. Hombergs, Breitbandiger Internetzugang durch Bitstromzugang, MMR-Beil. 2003, 37 (43). 
Anders sieht es etwa bei $\$ 6$ Abs. 1 GenTG aus. Zwar liegt mit dem Terminus des „neuesten wissenschaftlichen und technischen Kenntnisstand[s]“ ein explizit vager Begriff im oben genannten Sinne vor. Es ist allerdings von den Vertretern eines Beurteilungsspielraums ${ }^{81}$ nicht dargelegt, inwieweit sich Prozeduralisierung und Organisation des Wissens so strukturell etwa vom Immissionsschutzrecht unterscheiden, dass ein Spielraum darauf gegründet werden könnte. Gentechnik ist kein derart zusammenhängender gesellschaftlicher Prozess, so dass eine rekursive Vernetzung von Entscheidungen gegeben wäre. Es könnte aber ein Spielraum nach Typ 2 vorliegen: Die Kommission, die im Verfahren tätig wird, ist zwar ein Gremium von Sachverständigen, aber ohne systematisch eingebaute Perspektivenpluralität. Allerdings wirken nach $\mathbb{} 4$ Abs. 1 Nr. 2 GenTG einige Vertreter wichtiger gesellschaftlicher Gruppen mit; schließlich berät die Kommission nur und entscheidet nicht. Die Frage, ob sich daraus oder darüber hinaus in diesem Fall Gründe für einen Beurteilungsspielraum ergeben, kann hier nicht abschließend erörtert werden. Das Argument des für Regulierung nötigen impliziten Wissens greift jedenfalls nicht.

\section{Fazit}

Die Überlegungen zeigen, dass Gründe für eine normative Ermächtigung in den Strukturen der Wissensorganisation zu finden sind, mit denen die Verwaltung für bestimmte Entscheidungen ausgestattet ist. Der Bereich der Regulierungsverwaltung kann keineswegs in allen Entscheidungen Spielräume reklamieren, allerdings spricht die Kopplung von Regulierung an einen sozialen Prozess dafür, dass implizites Wissen des Regulierers in bestimmten Fällen für das Funktionieren der Regulierung konstitutiv sein kann. Jesch hat schon 1957 in einem Beitrag die Frage des Beurteilungsspielraums an die Mitteilbarkeit der entscheidungsrelevanten Informationen geknüpft - freilich noch ohne Regulierung einzubeziehen. ${ }^{82}$

Wo der Gesetzgeber den Grundsatz you only see when you know erkannt und in das Regulierungskonzept integriert hat, spricht dies für eine normative Ermächtigung der Verwaltung zur Letztentscheidung. Es erscheint gewinnbringend, andere Entscheidungstypen ebenfalls aus der Perspektive der Wissensorganisation zu analysieren und so die Dogmatik der Beurteilungsspielräume zu reformulieren.

Durch den Rekurs auf die Wissensperspektive lassen sich nicht nur Fallgruppen von Beurteilungsspielräumen genauer fassen, ein Vorteil kann auch die Anschlussfähigkeit im europäischen Kontext sein. Im europäischen Recht ist ein dogmatisch ein-

81 BVerwG NVwZ 1999, S. 1232 (1233 f.); OVG Berlin NVwZ 1995, S. 1023 (1024 f.); G. Beaucamp, Zum Beurteilungsspielraum im Gentechnikrecht, DÖV 2002, S. 24 (24 ff.); kritisch K.-H. Ladeur, Gefahrenabwehr und Risikovorsorge bei der Freisetzung von gentechnisch veränderten Organismen, NuR 1992, S. 254 (254).

82 D. Jesch, Unbestimmter Rechtsbegriff und Ermessen in rechtstheoretischer und verfassungsrechtlicher Sicht, AöR 82 (1957), S. 163 (223 ff.). 
deutiges Modell für die Begründung der Spielräume beim Normvollzug nicht festzustellen; jedenfalls hat sich keine an der deutschen Lehre orientierte Begründung durchgesetzt. Vielleicht kann eine Orientierung an Wissensverarbeitung eine Basis sein, zu einem mit anderen Rechtsordnungen kompatiblen Umgang mit Beurteilungsspielräumen zu kommen. 